\title{
Interpretations of the concepts of resilience and evolution in the philosophy of Leibniz
}

\author{
Vincenzo De Florio \\ The Global Brain Institute; Evolution, Complexity and COgnition research group \\ Vrije Universiteit Brussel, Brussels, Belgium \\ vincenzo.deflorio@gmail.com
}

V. 1.1, 2016-04-26.

\begin{abstract}
In this article I interpret resilience and evolution in view of the philosophy of Leibniz. First, I discuss resilience as a substance's or a monad's "quantity of essence" — its "degree of perfection" - which I express as the quality of the Whole with respect to the sum of the qualities of the Parts. Then I discuss evolution, which I interpret here as the autopoietic Principle that sets Itself in motion and creates all reality, including Itself. This Principle may be considered as a sort of ante-litteram metaphysical Darwinian Selection. My interpretations provide a different formulation for questions such as "why natural evolution evolves?" and "why is this the best of all possible worlds?" In this article I also provide a geometrical interpretation of a key aspect behind the "divine mathematics" of the autopoietic Principle.
\end{abstract}

\section{Introduction}

In all things there are different quantities of essence - different degrees of self-determination. And every concept associated to a thing shouts its uniqueness, proclaims its peculiarity, its right to exist and be persisted. Against all odds. That is resilience: actively pursuing one's right to existence. A fair assessment of this right can only occur by means of a fair verification of the ability to persist and co-exist. A metaphysical attraction drives a natural selection process among the current material manifestations (that is, reifications) of the Ideas. Said Metaphysical Mechanics continuously refines those reifications, making them closer and closer to their ideal counterpart. It's what we call evolution. Even this process is an Idea - an autopoietic principle whose reification is the current evolution state of the world. As it is the case for all reifications, also the reification of this principle tends to perfection, which sets itself in motion and produces all reality, which by necessity is the best of all possible worlds.

\section{Resilience and Evolution}

\section{Resilience}

Resilience is a system's ability to strive to persist its identity. In other words, resilience is entelechy - namely "being-at-work to stay-the-same" (Sachs, 1995). In my works, and 
especially in (De Florio, 2015a; De Florio, 2015b), I observe how a "system" may be resilient in two ways: intrinsically and extrinsically.

Intrinsic resilience: a system may be called "in itself" resilient: resilient, because of its own "design". When we say that a system of the class "Man" is characterized by superior resilience with respect to any other system of the class "Animal" we are talking of intrinsic resilience, namely we are considering the design characteristics of two classes of systems. We are in fact discussing two ideas; or we could say in technological terms two blueprints; or, in philosophical terms, two Monads. Resilience is in this case the quality of the Whole with respect to the sum of the qualities of the Parts (De Florio, 2016a).

For some still unfathomed mechanism, nature selects their Substances by rewarding those that implement —albeit clumsily — the most resilient and most "evolved" ideas. Said Darwinian Metaphysical Selection is associated to a second aspect of resilience:

Extrinsic resilience: apart from being resilient-by-design, a system may also be resilient with respect to the environment it is set to operate in; and, in particular, a system may be resilient with respect to the other "clumsy implementations" with which that system shares a finite environment - the world. Thus it is resilience also the outcome of the match between every implementation (in Leibnitian terms, any Substance) and a random series of dynamically changing situations. The (intrinsic) blueprint is thus evaluated by matching its current implementation with the mutating manifestations of a dynamic environment with the terminology of dynamic systems, its orbits. Said match "breaks down" the unity of the Whole into the multiplicity of its Parts; and it reveals how some of the design-specific and of the implementational-specific aspects are more suitable, and thus more desirable, than others. If an individual or a species is endowed with a "winning combination" of the winning aspects, then it will survive; and with it, each of the winning aspects will also survive, by "bonding" itself to one or more other winning aspects. This way, winning blueprints persist their existence. Quoting Leibniz,

"Essence in itself strives for existence. From this it follows further that all possible things, i.e. things expressing an essence or possible reality, strive with equal right for existence in proportion to their quantity of essence or reality, or to the degree of perfection which they contain; for perfection is nothing other than quantity of essence.

Hence it is very clearly understood that out of the infinite combinations of possibles, and possible series, there exists one through which the greatest amount of essence or possibility is brought into existence. [...] The situation is like that in certain games where all the spaces on the board are to be filled according to certain rules, and where, unless you use some skill, you will in the end be excluded from certain spaces and forced to leave more spaces empty than you could have or wished to. But there is a definite rule through which the maximum number of spaces is most easily filled. [...] In short it is just like tiles that are arranged so that as many as possible occupy a given area.
A certain divine mathematics or metaphysical mechanics is employed in the very origination of things." (Leibniz, 1697)

Intrinsic and extrinsic resilience are very different from each other. I mentioned how "Man" is intrinsically superior to "Dog"; and yet, let us suppose that we have a man and a dog in 
an environment in which a threat is announced by ultrasonic noise. In such an environment it is the dog (or, better, its auditive blueprint) that "wins" over the man (or better, his auditive blueprint). In this particular confrontation of ideas, in which the rules are set by the environment, it is the dog that exhibits the highest (extrinsic) resilience.

A direct consequence of the above discussion is my stance, introduced in draft paper (De Florio, 2015c), that resilience should be discussed in terms of Game Theory - as a set of concurrent confrontations taking place across all of the system's scales. In order to represent such multi-scale dynamics, in the cited paper I proposed to model systems as nested compositional hierarchies and model extrinsic resilience via interconnected and mutually influencing payoff matrices (which I called as payoff "spreadsheets"). The solution of said payoff spreadsheets would represent the overall outcome of all the concurrent interplays between a system's organs and Parts and a "shock" produced by the environment.

I feel it important to remark also how the world's finiteness is an important "ingredient" of the Darwinian Metaphysical Selection introduced by Leibniz. In Leibniz, finiteness is not an imperfection; rather, it is one of the key "ingredients" of his metaphysical mechanics. Finiteness introduces the constraints through which competition arises between substances - or, in other words, the competition that arises between the coeval material implementations of the Ideas. Leibnitian concept of compossibility manifests itself only when resources are limited - for instance, when the predominance of a class of substances (for instance, a species) impoverishes beyond the limits of survival the living space of another class of substances.

My papers (De Florio, 2014a; De Florio, 2016b) provide more information of my models of resilience and their relation with philosophy of science and technology - as well as with modern science and technology. Concepts such as resilience; system; natural selection; pray-predator relationships; cellular automata; artificial life; virtual reality; and biological evolution, are but a few of the supposedly "modern" concepts whose foundation lies in the Philosophical Systems of Aristotle, Plato, and especially Leibniz.

\section{Evolution}

A fundamental scientific and philosophical question that I have been addressing time and again is the one introduced in (Wagner \& Altenberg, 1996):

Why evolution evolves? What are the reasons that lead natural evolution to evolve itself? Why does nature produce ever more "evolved" substances?

It is important to note how such a question is multidisciplinary in nature, as it requires contribution from as different a field as computer science, biology, philosophy of science and technology, mathematics, network theory, microeconomy, and ecology - to name but a few. For instance,

- From a philosophical point of view, the problem can be reformulated as follows: what are the conditions that lead the "distance" between a substance and its Monad to decrease monotonically, at least for a non-empty set of substances? 
- From a mathematical point of view, the problem may be expressed as a statement about the convergence of the substances' quantity of essence: let us call QoE the quantity of essence that characterizes monads and substances, and $s(t, m)$ the substance implementing Monad $m$ at time $t$. Let us suppose that a non-empty set of substances exist at $t=0$. Then what are the conditions that lead quantity

$$
\mathrm{QoE}(m)-\mathrm{QoE}(s(t, m))
$$

to tend to zero for $t$ increasing indefinitely, $\forall m \in M \neq \varnothing$ ?

- From a computer science point of view: let us consider an evolutionary algorithm that operates Darwinian selection on a set of agents or genes constrained to a limited world and to trophic (predator-prey) relationships. What are the conditions that lead the algorithm to identify a non-empty set of "best-resilient" agents/genes?

It is again Leibniz the author that expressed this problem and possible solutions with the highest clarity of representation - if you allow me the pun. It is indeed the best of all possible worlds the one we are in, precisely because of the algorithm that rules it is steadily converging towards the sought solutions. Implementations are becoming more and more perfect, more and more ideal one could say, and God's promise is that, at the end of times, the chosen substances shall touch their Monad. They shall become their Monads; transcend into them. Leibniz tells us that each Monad is the unmoved mover (Aristotle, 1999) of its material substance, and that the Ultimate Monad is the unmoved mover of the processes that constitute (actually, realize!) the Universe. Thus this is the already mentioned "divine mathematics or metaphysical mechanics" that "is employed in the very origination of things" (Leibniz, 1697): an autopoietic Principle, which sets Itself in motion and creates all reality, including Itself.

And yet this Grand Motion appears to originate from simpler, tinier principles and small movements. "Per parva ad astra", Leibniz seems to suggest: the great things are reached through small steps, and pertain to elements and principles that are far from complicated. It is the Ars Combinatorica: a "method" that steadily refines more and more complex structures from simple initial representations. Structures not just complex, but modular in nature.

An example of this simple combinatorial refinement process leading to complex structures can be found in my research on so-called fractal social organizations (De Florio et al., 2013): 


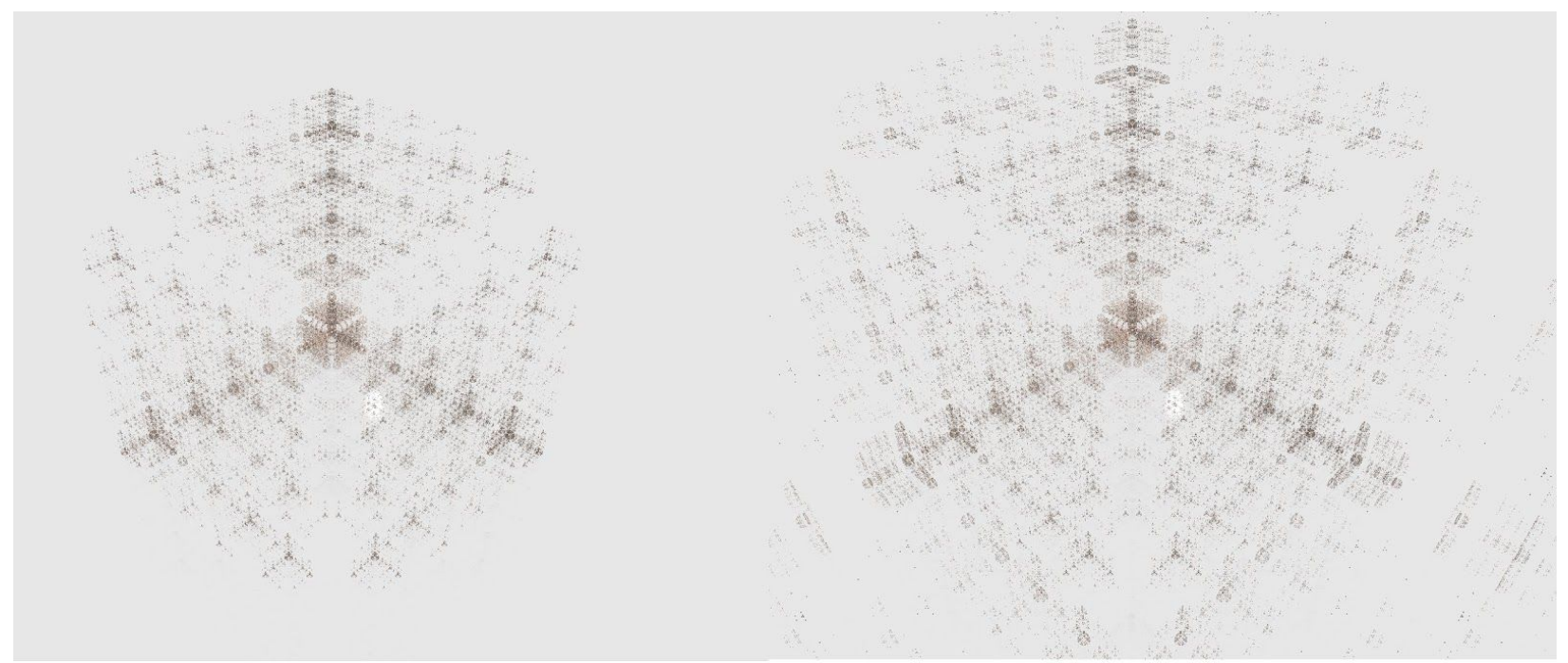

Figure 1: Combinatorial development of 000000012345 (left-hand side) and 000000012345. Similar images are obtained with seeds in the form 0n12345, $n$ odd. Intuitively, seeds 0n12345 all "converge" to the same concept -- a concept which becomes more and more delineated and clear... Though the "real" concept all those strings hint is not here, it is not fully representable: it is the concept that one gets for $n$ going to infinity. What we can touch is not the real thing...

The left-hand side image in Figure 1 is a combinatorial development that derives from seed "000000012345" (a "gene" made of twelve "bases" belonging to six classes, identified by integers $0, \ldots, 5$, with class 0 including seven elements and classes 1 -to- 5 including just one element). The process that derives the image from the seed is omitted here for the sake of brevity - please refer to (De Florio, 2005) for a detailed description. We could say that that image is the "phenotype" produced by that process starting from gene 000000012345 . Now, by looking at the left-hand side image we can observe two things: first, that the process develops modularity: it is possible to identify "blocks". And second, those blocks are phenotypes of sub-genes of 000000012345 ! In other words, the geno-pheno process preserves the property of modularity ${ }^{1}$.

This can be seen more clearly by considering the right-hand side image in Figure 1, which shows a portion of the phenotypical development produced by gene 000000000012345 . As the first gene is a sub-string of the second gene, so the phenotype derived from the first gene is a sub-image of the image produced by the second gene.

Figure 2 shows the whole phenotypic expansion of the second gene:

${ }^{1}$ Remarkably enough, modularity has been identified as pne of the preconditions to evolvability (Hansen, 2003; Wagner \& Altenberg, 1996). 


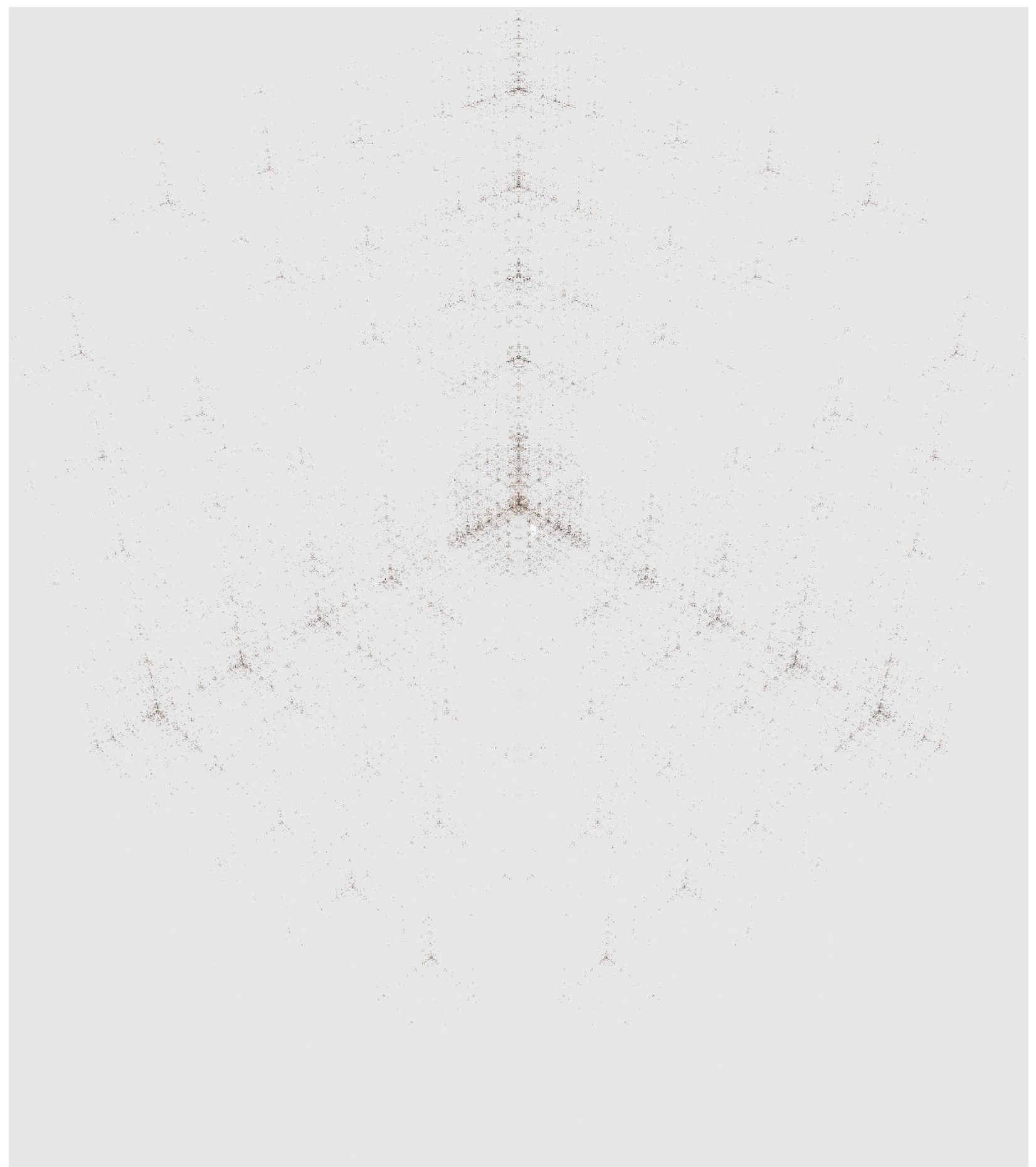

Figure 2: Phenotypical expansion of gene 000000000012345.

Images such as the ones above provide a geometrical representation of the quantity of essence. Given any concept, its representation includes modules. Said modules are nothing but the representations of the sub-concepts that are necessary to define that concept. In other words, they are pictograms that are needed to compose a grander pictogram. This closely correspond to Leibniz's Characteristica Universalis. Each 
pictogram is at the same time a conceptual Whole and a set of Parts; it can be considered as a single fact or as the combination of sub-facts that lead to the truth of the Whole. It is a module but it is also the set of "instructions" that constitute and justify it, realize it. It is the means we can reason about a substance's intrinsic resilience via some mechanical diagrammatic reasoning process.

Is possibly that the reason why Leibniz considered so highly the theory of preformationism by Antonie Van Leeuwenhoek? Was it maybe because preformationism exemplified, albeit in an unscientific way, the very concept of conservation of modularity when passing from a genotypical representation (viz. a concept, namely an abstract and general blueprint) to a phenotypical representation (namely a particular "realization", or concrete expansion, of that template)? I stated my stance on this matter in (De Florio, 2014):

"This property [of conservation of modularity], which may be probably best represented through the mathematical concept of an isomorphism between genotypical and phenotypical algebraic domains, is in fact compatible with the Leibnitian vision of substances as "second-order scripts" produced by "first-order scripts". As already mentioned, this conservation of modularity possibly hints at the "greater secret" mentioned by Leibniz, namely the reasons why evolution "evolves", and why nature "naturally" develops ever more complex substances."

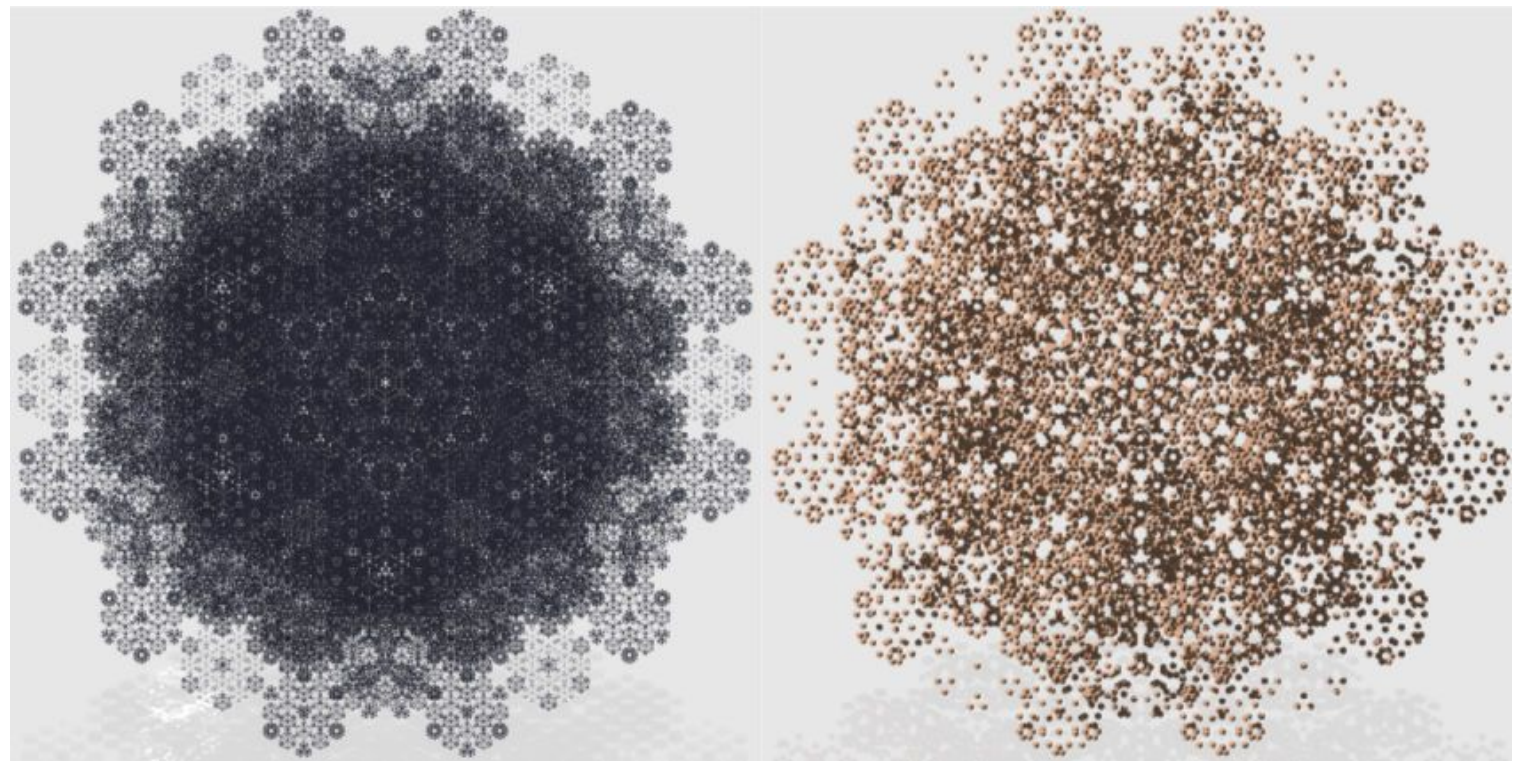

Figure 3: Another example of conservation of modularity. Left-hand image is the phenotic expansion of gene 011112233334 . The right-hand image is the phenotypic expansion of 011123334.

\section{Conclusions}

Leibniz's philosophy arrived to us in a very fragmented way, scattered through a large number of small documents. Those many documents revel the strain towards a unitary vision able to capture the complexity of the all. The complexity of describing said vision could possibly be eased by considering a number of interrelated themes. In this paper I propose to consider two such intertwined Gestalts, namely resilience and evolution. I 
associate the concept of resilience to that of quantity of essence (a system's degree of perfection). I show how evaluating resilience calls for the evaluation of a substance-environment fit, which corresponds to the Leibnitian concept of compossibility. Later on, I associate the concept of evolution with the metaphysical mechanics that is in Leibniz at the root of the very origination of things. I show how modern questions of the philosophy of biology, such as "why natural evolution evolves?", can be reinterpreted, e.g., as convergence statements on the quality of essence of mathematical or computer-based entities. Although Leibniz was obviously not aware of any theory of natural evolution, it is tempting to define his metaphysical mechanics as a sort of Darwinian Metaphysical Selection. It is possibly the apparent effectiveness of said mechanism that led Leibniz to consider this as the best of all possible worlds.

I conclude this work by discussing the nature of Leibniz's divine mathematics. By means of a simple combinatorial example, I provide a geometrical representation of the quantity of essence. Given any concept or cause, its representation includes modules. Said modules are nothing but the representations of the sub-concepts that are necessary to define the root concept, or root cause. In other words, they are pictograms that are needed to compose a grander pictogram. This closely correspond to Leibniz's Characteristica Universalis.

\section{References}

(Aristotle, 1999) Aristotle. Metaphysics, Joe Sachs (trans.), Green Lion Press, 1999. (De Florio et al., 2013) Vincenzo De Florio, Mohamed Bakhouya, Antonio Coronato, and Giovanna Di Marzo. Models and Concepts for Socio-technical Complex Systems: Towards Fractal Social Organizations. Systems Research and Behavioral Science, Vol. 30, No. 6, Nov.-Dic. 2013, pp. 750-772. DOI: 10.1002/sres.2242.

(De Florio, 2005) Vincenzo De Florio. Permutation Numbers. Complex Systems, Vol. 15, No. 2 (2005). Complex Systems Publications, Champaign, IL. 24 pages.

(De Florio, 2014) Vincenzo De Florio. Behavior, Organization, Substance: Three Gestalts of General Systems Theory. In Proc. of the IEEE 2014 Conference on Norbert Wiener in the 21st Century. Boston, MA, 24-26 June 2014.

(De Florio, 2015a) Vincenzo De Florio. On resilient behaviors in computational systems and environments. Journal of Reliable Intelligent Environments, Vol. 1, No. 1. Springer. DOI: $10.1007 / \mathrm{s} 40860-015-0002-6$.

(De Florio, 2015b) Vincenzo De Florio. On environments as systemic exoskeletons: crosscutting optimizers and antifragility enablers. Journal of Reliable Intelligent Environments, Vol. 1, No. 2. Springer. DOI: 10.1007/s40860-015-0006-2.

(De Florio, 2015c) Vincenzo De Florio. Resilience as concurrent interplays of opponents: preliminary ideas and call for collaborations. Posted on ERACLIOS at http://eraclios.blogspot.be/2015/10/resilience-as-concurrent-interplays-of.ht $\underline{\mathrm{ml}}$.

(De Florio, 2016a) Vincenzo De Florio. On the quality of emergence in complex collective systems. To appear in Mohammad Essaaidi, Mohamed Nemiche (Eds.), "Advances in Complex Societal, Environmental and Engineered Systems", Springer series "Nonlinear Systems and Complexity", Springer, 2016.

(De Florio, 2016b) Vincenzo De Florio. Systems, Resilience, and Organization: Analogies and Points of Contact with Hierarchy Theory. CoRR, Vol. abs/1411.0092. 
http://arxiv.org/abs/1411.0092.

(Hansen, 2003) Thomas F. Hansen. Is modularity necessary for evolvability? Remarks on the relationship between pleiotropy and evolvability. BioSystems, Vol. 69 No. 2 (2003), pp. 83-94.

(Leibniz, 1697) Gottfried Wilhelm von Leibniz. Die philosophischen Schriften von Gottfried Wilhelm Leibniz. C. I. Gerhardt (Ed.), Vol. VII, Berlin: Weidmann, 1875-90. In L. H. Strickland (2006), The shorter Leibniz texts: a collection of new translations. Continuum. ISBN 9780826489517.

(Sachs, 1995) Joe Sachs. Aristotle's Physics: A Guided Study. Masterworks of Discovery, Rutgers University Press. ISBN 0-8135-2192-0.

(Wagner \& Altenberg, 1996) Gunter P. Wagner and Lee Altenberg. Complex Adaptations and the Evolution of Evolvability. Evolution, Vol. 50, No. 3 (June 1996), pp. 967-976. Society for the Study of Evolution. 